\title{
Modelling of Plant Growth with Apical or Basal Meristem
}

\author{
N. Bessonov ${ }^{1}$, F. Crauste ${ }^{2}$, V. Volpert ${ }^{2 *}$ \\ ${ }^{1}$ Institute of Mechanical Engineering Problems, 199178 Saint Petersburg, Russia \\ ${ }^{2}$ Institut Camille Jordan, UMR 5208 CNRS, University Lyon 1 \\ 69622 Villeurbanne, France
}

Dedicated to the memory of Loïc Forest

\begin{abstract}
Plant growth occurs due to cell proliferation in the meristem. We model the case of apical meristem specific for branch growth and the case of basal meristem specific for bulbous plants and grass. In the case of apical growth, our model allows us to describe the variety of plant forms and lifetimes, endogenous rhythms and apical domination. In the case of basal growth, the spatial structure, which corresponds to the appearance of leaves, results from dissipative instability of the homogeneous in space solution. We study nonlinear dynamics and wave propagation of the corresponding reaction-diffusion systems. Bifurcation of periodic at infinity waves is investigated numerically.
\end{abstract}

Key words: plant growth, basal meristem, dissipative structures, travelling waves AMS subject classification: $92 \mathrm{C} 80$

\section{Models of plant growth}

One of the most important features of plants, for purposes of modelling them, is that proliferating cells are strongly localized. The growing part of the plant where cells divide is called the meristem. The primary or apical meristem is located at the very end of growing shoots and represents a narrow layer of cells with a more or less constant width for each particular plant. The biological

*Corresponding author. E-mail: volpert@math.univ-lyon1.fr 
mechanism that provides the localization of the apical meristem is related to the expression of certain genes.

Some parts of the primary meristem can remain in the internodes. Under certain conditions, determined by plant hormones, they can lead to the appearance of buds that can develop into branches. The secondary meristem, or cambium, is responsible for width-wise growth of the plant.

If we consider only the apical meristem, then we can say that cell proliferation and growth determine plant growth. Outside this narrow layer, cells differentiate; they cannot divide any more, and they serve to conduct biological products. Cell division and growth are controlled by external signals called growth and mitosis factors. Each of them is a generic name for a number of biological products. In particular, mitosis factors indicate the cell whether it should go from a rest state, where it can remain an indefinitely long time, to a division cycle. In some cases, the same molecule can play both roles. In what follows we will not distinguish between these two factors and will call them for brevity GM-factors. They are produced in meristemic cells and can be transmitted between neighboring cells. A detailed introduction of plant growth biology is presented in the first paper of this issue [6]. So we restrict ourselves here to a short description of biological observations, which we use in this work.

Studies of plant growth probably begin from ancient times. In the middle ages, Leonardo da Vinci observed the seasonal periodicity of growth and some features of plant forms ([7], pages 239, 912). Theories of phyllotaxis, which can be defined as a construction determined by organs, parts of organs, or primordia of plants ([11], page 286) appear already in the XVII-th century. D'Arcy Thompson reviewed early theories ([7], Chapter XIV) and R.V. Jean contemporary theories of phyllotaxis [11]. An important development received algorithmic approaches to plant modelling where some elementary units (branch, bud, flower) are assembled according to some formal rules (see [11], [13], [18] and the references therein).

Another possible approach to plant growth modelling is based on the description of biological mechanisms of phyllotaxis. These mechanisms can be related to Turing structures with long range inhibition and short range activation ([15], [16] and the references therein). This mechanism of pattern formation is widely used in mathematical biology even if it is sometimes difficult to determine the underlying molecular mechanism. More recent development in plant growth biology and modelling takes into account the role of auxin transport and distribution in phyllotaxis [12], [17], [20], [23] and in radial growth of branches [8].

In this work we attempt to take into account biological mechanisms of phyllotaxis together with fluxes of nutrients and metabolites in the whole plant. Relatively simple structure of plants, where the growing part is strongly localized, suggests very natural mathematical models describing their growth. In [2], [3] plant growth is described with free boundary problems where the motion of the interface corresponds to the displacement of the apical meristem. The speed of growth, that is of the interface motion, is determined by diffusion and convective fluxes of nutrients in the plant and by a self-accelerating production of plant growth factors in the meristem.

GM-factor production is self-accelerating. This property determines the specific form of GMfactor production rate (function $g$ in Section 2), which depends on the GM-factor concentration itself. This concentration must be seen as a marker of the progression through cell cycle. In the first part of the cycle (G1 - S), GM-factor concentration can be related to the concentration of 
transcription factor $E 2 F$, which determines (together with RB protein) the transition from the $G_{1}$ phase to the $S$ phase, enabling the consequent cell proliferation [14], [22]. During the second part of the cycle (G2 - M), GM-factor concentration is related to the complex cyclinB-cdc2, controlling the process of mitosis. Since the production of cyclinB-cdc 2 complex is self-accelerating through the activation by cdc25, we can then consider that production of GM-factor is self-accelerating. Cell cycle can also be influenced by auxin in its first part and by cytokinin in the second [5], [9], [14], [21], [22]. Throughout this manuscript, GM-factor concentration will be denoted by $R$.

The rate of production of the GM-factor depends also on the concentration of nutrients, which can be phosphorus and nitrogen salts, supplied from the roots, or sugars (mostly sucrose) produced in the process of photosynthesis in all green parts of the plant. We will not specify here the origin of nutrients coming to the apical meristem from lower parts of the plant and will denote their concentration by $C$. The concentration of the GM-factor determines the proliferation rate of cells in the meristem and, consequently, the rate of growth.

In Section 2 we will discuss growth by apical meristem. We will describe endogenous rhythms, branching patterns, variety of forms and lifetime of plants. Section 3 is devoted to growth by basal meristem. Its main difference from the apical meristem, from the modelling point of view, is that it does not change its location in the process of plant growth. Cell proliferation in the meristem results in leaf growth. As above, the model includes the equations for nutrients $C$ and for the GM-factor $R$. The particular form of the equations takes into account their production and consumption in the basal meristem. Leaf formation and growth corresponds to the emergence of a nonhomogeneous spatial distribution of $C$ and $R$ in the basal meristem. This distribution results from the Turing instability of a homogeneous in space solution. Turing structures arise in various problems of morphogenesis [16] including phyllotaxis [15]. They are observed in the presence of two species conventionally called activator and inhibitor. The mechanism of structure formation suggested in this work in order to describe leave formation by basal meristem is different. It is based on the interaction of self-accelerating production of the GM-factor and of nutrient consumption.

In Section 4, we numerically investigate the bifurcation of waves periodic at infinity, for the model of plant growth with basal meristem, developed in Section 3.

\section{Growth by apical meristem}

\subsection{D model}

If the plant grows due to cell proliferation in the apical meristem, then we can describe its growth by a free boundary problem where the free boundary corresponds to the apical meristem and its displacement to plant growth. Such models are developed and studied in [2]-[4]. The simplest onedimensional model where the plant is represented by an interval whose length depends on time has the form

$$
\begin{gathered}
\frac{\partial C}{\partial t}+u \frac{\partial C}{\partial x}=d \frac{\partial^{2} C}{\partial x^{2}}, \\
h \frac{d R}{d t}=Z(A, Q) g(R) C-\sigma R,
\end{gathered}
$$


where $0 \leq x \leq L(t), L(t)$ is the shoot length, $C$ is the concentration of nutrients coming from the root, $u$ is the convective speed of nutrients determined from the continuity equation for the incompressible fluid and related to the speed of growth of the interval,

$$
u=\frac{d L}{d t}
$$

The concentration of growth and mitosis factor $R$ is defined at the growing end $x=L(t)$ which corresponds to the apical meristem. Equation (2.1) describes diffusive and convective transport of nutrients through the plant, equation (2.2) describes the production and consumption or destruction of the GM-factor in the apical meristem. Here $h$ is a parameter (the width of the meristem), the function $Z(A, Q)$ describes the dependence of the rate of the production of the GM-factor on plant hormones auxin $A$ and cytokinin $Q$. The plant growth rate depends on $R$,

$$
\frac{d L}{d t}=f(R)
$$

The specific forms of the functions $f(R)$ and $g(R)$ are described below.

The one-dimensional model is justified if the length (or height) $L$ of the plant is essentially greater than the diameter of its trunk. Hence we consider the interval $0 \leq x \leq L(t)$ with the length depending on time. The left endpoint $x=0$ corresponds to the root. Its role is to provide the flux of nutrients taken into account through the boundary condition. We do not model the root growth here. Therefore the left boundary is fixed. The right endpoint, $x=L(t)$ corresponds to the apex. Its width is much less than that of the plant. We suppose in the model that it is a mathematical point. The value $L(t)$ increases over time. According to the assumption above, the growth rate is determined by the concentration of metabolites at $x=L(t)$, equation (2.4).

System (2.1), (2.2) should be completed by the boundary conditions for $C$ at $x=0$ (supply of nutrients) and at $x=L(t)$ (flux of nutrients to the meristem):

$$
x=0: C=C_{0}, \quad x=L(t): d \frac{\partial C}{\partial x}=-g(R) C .
$$

The second boundary condition shows that the flux of nutrients from the main body of the plant to the meristem is proportional to the concentration $C(L, t)$. This is a conventional relation for mass exchange at the boundary, called Robin boundary conditions. The factor $g(R)$ shows that this flux can be regulated by proliferating cells.

We recall that the interval $0<x<L(t)$ corresponds to differentiated cells that conduct nutrients from the root to the apex. We suppose that they are in a liquid solution.

System of equations (2.1)-(2.5) is a generic one-dimensional model of plant growth based on: a) "continuous medium" assumptions of mass conservation (for $C+R$ ) and of the proportionality of the flux $\partial C / \partial x$ at the boundary to the value of $C$; and b) a "biological" assumption that there is a chemical species $R$, the plant growth and mitosis factor, which is produced in the meristem and which determines the plant growth.

We note that the conservation of mass in the case $\sigma=0$ implies that the term $g(R) C$ enters both the boundary condition and equation (2.2). Therefore, the assumption that the rate of the plant 
growth factor production depends on its concentration $R$ makes the boundary condition depend on it also. Properties of the function $g$ can be crucial for plant growth. In particular, if it is constant (the production rate is not auto-catalytic), the model does not describe the essential difference in plant sizes [3].

We now specify the form of the functions $f$ and $g$. We will consider $f$ as a piece-wise constant function equal to 0 if $R$ is less than a critical value $R_{f}$ and equal to some positive constant $f_{0}$ if $R$ is greater than $R_{f}$ (Figure 1a). This means that growth begins if the concentration of the plant growth factor exceeds some critical value.
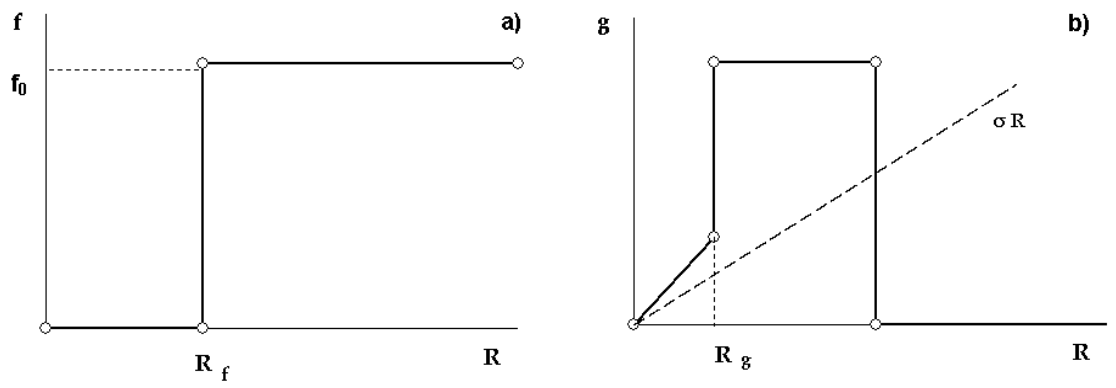

Figure 1: Functions $f$ and $g$.

The production of the growth factor $R$ is assumed to be auto-catalytic. For the sake of simplicity, we consider a piece-wise linear function $g(R)$ (Figure 1b). In some cases smooth functions $f$ and $g$ can also be considered.

These assumptions are consistent with plant morphogenesis. It is well known, for example, that auxin, produced in the apex, stimulates mitosis and cell proliferation. An other hormone, kinetin, is also known to stimulate cell proliferation. Production of mitosis factors can be self-accelerating through cyclinB-cdc2 complex and activation by cdc25 [10]. For large concentrations, the cyclin can also inhibit its own production ([1], p. 876).

An example of numerical simulations of problem (2.1)-(2.5) is shown in Figure 2. In this case growth is periodic in time (Figure 2a). Short periods of growth are separated by long time intervals where the length does not change. The length change is approximately the same during all periods of growth except for the first one, where it is about two times greater. The periods without growth become longer over time. This is related to the growing length of the interval. For larger $L$ it takes more time for the concentration $C(L, t)$ to become large enough for $R(t)$ to increase. Figure $2 \mathrm{~b}$ shows the function $R(t)$. These oscillations can be related to endogenous rhythms in plants. Another mode of growth, where the length changes linearly in time until growth stops, is observed for other values of parameters [3]. 

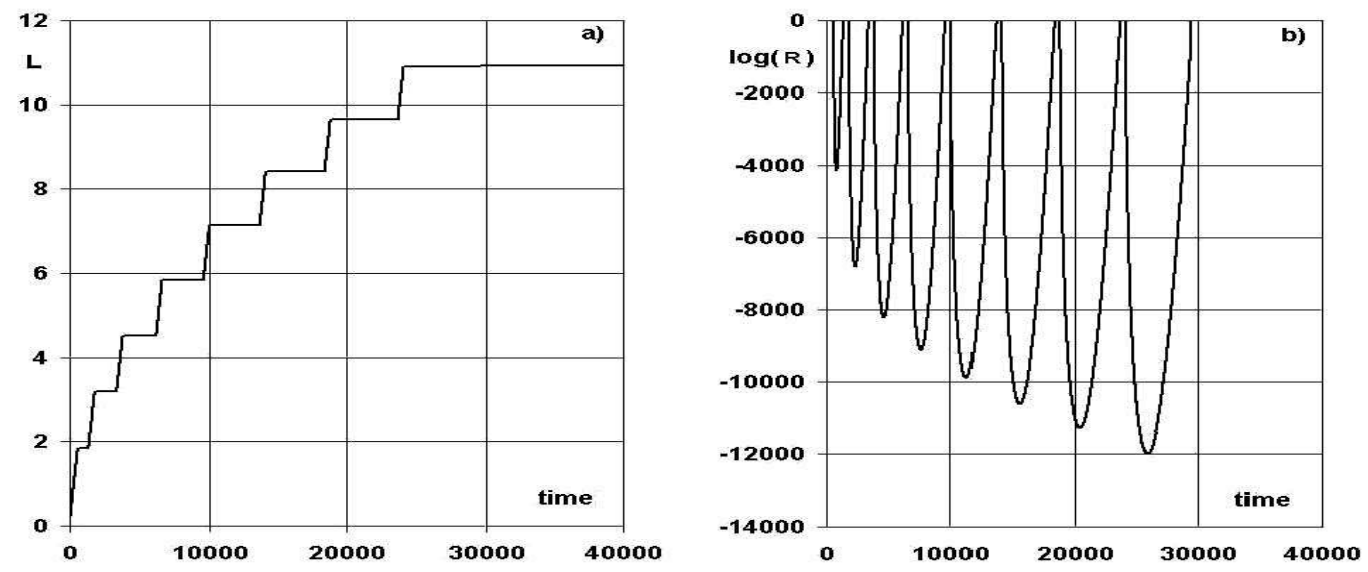

Figure 2: Length of the plant (left) and the value of mitosis factor at the meristem (right) as functions of time.

\subsection{D model with branching}

In this section we discuss conditions of appearance of branches in the framework of the onedimensional model of plant growth. We use the experimental observation on shoot and root growth from calus: if the concentrations of two hormones, auxin and cytokinin (which we denote by $A$ and $Q$, respectively) are in a certain proportion, then shoots will appear. For a different proportion, no shoots but rather roots will grow [19], [25].

Hormone $A$ is produced in growing parts of the plant (leaves, shoots); hormone $Q$ either in roots or in growing parts. In our model, $A$ will be produced at the moving boundary $x=L$ that corresponds to the apical meristem. The rate of production is proportional to the growth rate. Hormone $Q$ will either be supplied solely through the stationary end of the interval $x=0$ (the root) or will also be produced at the moving boundary.

The concentrations of nutrients $C$, and of hormones $A$ and $Q$ are described by the diffusion equations with convective terms:

$$
\begin{aligned}
& \frac{\partial C}{\partial t}+V \frac{\partial C}{\partial x}=d_{C} \frac{\partial^{2} C}{\partial x^{2}}-\gamma C, \\
& \frac{\partial Q}{\partial t}-V_{Q} \frac{\partial Q}{\partial x}=d_{Q} \frac{\partial^{2} Q}{\partial x^{2}}-\mu Q \\
& \frac{\partial A}{\partial t}-V_{A} \frac{\partial A}{\partial x}=d_{A} \frac{\partial^{2} A}{\partial x^{2}}-\mu A
\end{aligned}
$$

The convective speed $V$ in the first equation is determined as the speed of growth:

$$
\frac{d L}{d t}=V, \quad V=f(R)
$$


Here $d_{C}, d_{Q}, d_{A}$ and $\mu$ are parameters; the space variable $x$ is defined independently for each branch. The convective speeds $V_{A}$ and $V_{Q}$ in equations (2.7) and (2.8) can be different in comparison with equation (2.6). It corresponds to transport in the phloem in the direction from top (meristem) to bottom (root). The last term in the right-hand side of equation (2.6) describes a possible consumption of nutrients along the stem. This assumption is biologically relevant. We did not consider it in the previous section in order to simplify the basic model.

The rate of production of the GM-factor at $x=L(t)$ is given by the equation

$$
h \frac{d R}{d t}=Z(A, Q) g(R) C-\sigma R .
$$

Here $Z(A, Q)=Z_{1}(A) Z_{2}(Q)$

$$
Z_{1}(A)=\left\{\begin{array}{cc}
k A, & 0 \leq A \leq 1 / k \\
1, & 1 / k \leq A \leq A_{0}-1 / k \\
k\left(A_{0}-A\right), & A_{0}-1 / k \leq A \leq A_{0} \\
0, & A_{0} \leq A
\end{array}\right.
$$

and the function $Z_{2}(Q)$ is defined similarly. The form of the functions $Z_{1}(A)$ and $Z_{2}(Q)$ is chosen in accordance with the biological observations that there are optimal concentrations of plant hormones. Proliferation of plant cells can decelerate if the concentrations are too low or too high. In particular, it is the case of auxin playing an important role in apical domination.

The initial and boundary conditions for $L, R$ and $C$ are the same as for the 1D model without branching (Section 2.1), the concentrations $A$ and $Q$ are initially zero. The boundary conditions for $Q$ describe its possible production in the root, and its production in the meristem with rate proportional to the rate of growth:

$$
\left.Q\right|_{x=0}=Q_{0},\left.\quad d_{Q} \frac{\partial Q}{\partial x}\right|_{x=L}=\varepsilon V .
$$

Finally, the boundary conditions for $A$ are similar, except that the boundary condition at $x=0$ takes into account that this hormone can be transported from the stem to the root:

$$
\frac{\partial A}{\partial x}-\left.\beta A\right|_{x=0}=0,\left.\quad d_{A} \frac{\partial A}{\partial x}\right|_{x=L}=\varepsilon V .
$$

We define next the branching conditions. A new branch appears at $x=x_{0}$ and $t=t_{0}$ if

$$
A\left(x_{0}, t_{0}\right)=A_{b}, \quad Q\left(x_{0}, t_{0}\right)=Q_{b},
$$

where $A_{b}$ and $Q_{b}$ are some given values. Appearance of a new branch means that there is an additional interval connected to the previous one at its point $x_{0}$. The variables $C_{n}, A_{n}, Q_{n}$ and $R_{n}$ are described at the new interval by the same equations as above. Here the subscript $n$ determines the number of the branch. We should complete the formulation by the initial value of the concentration $R_{n}=R_{n}\left(t_{0}\right)$. It cannot be found as a solution of the problem but should be considered as a parameter. 
Formation of a new branch is accompanied by production of $A$ and $Q$. It is not described by equations (2.7) and (2.8). It is an additional source term localized in the vicinity of each point $x_{0}$ where condition (2.13) is satisfied during a short time after $t=t_{0}$. Therefore, condition (2.13) will not be satisfied in the vicinity of the new branch any longer, and the set of branch will be discrete. To complete the model we impose additional conditions at the branching points: continuity of concentrations and conservation of fluxes.

The branching conditions are formulated above in terms of plant hormones, auxin and cytokinin. In reality the role of these hormones is essentially more complicated and not completely understood. In particular, it is known that auxin plays an important role in formation of plant organs and in plant diversity.

We recall that the local growth rate of the apical meristem is determined by the function $f(R)$, which is characterized by two parameters $R_{f}$ and $f_{0}$ (see Figure 1 a). The first one determines the critical value of the GM-factor for which the plant growth begins. The second one determines the rate of growth. Both of them can influence the growth pattern. In the $1 \mathrm{D}$ model without branching, the value of $R_{f}$ determines the mode of growth, oscillating or linear. Based on these considerations, we can suppose that $R_{f}$ and $f_{0}$ may depend on the auxin concentration.

A typical example of plant growth in the 1D model with branching is shown in Figure 3 . In the beginning of the evolution there is a single branch which grows with an approximately constant speed until the growth period is finished. The apical meristem is located at the upper end of the interval. The plant hormones auxin and cytokinin are produced there and are transported along the whole branch. It can be diffusive or convective transport. Cytokinin can also be produced in the roots. This is taken into account through the boundary condition at $x=0$.

If at some point of the branch the concentrations of auxin and of cytokinin take on some prescribed values, then a new bud appears. In the simulation shown in Figure 3, there are five buds that appear one after another at an approximately equal distance. Each of the buds contains its own apical meristem with some value of the GM-factor $R$. When a new bud appears, the initial value $R_{0}$ is prescribed. It can be some given constant or it can depend on some factors (on plant hormones or on the value of $R$ in the apical meristem of the main growing branch). After that, the value of $R_{i}$ in the bud evolves according to the same equation (see (2.10)).

When $R_{i}$ becomes larger than $R_{f}$, (see (2.9) and Figure 1), proliferation begins and a new branch starts growing from the bud. When the main branch stops growing, under appropriate conditions a new branch can appear from another bud. This is related to apical domination: growth of main branch suppresses growth of other branches.

As it is discussed above, we consider diffusive and convective fluxes of nutrients. Diffusive flux acts throughout all branches, whereas convective flux is directed to growing branches. This is related to the continuity equation for the incompressible fluid. The routes of convective flux of nutrients is shown in red in Figure 3.

Thus, initiation of growth of new branches is determined by the interplay between the concentrations of nutrients and of the growth factor. In the example presented in Figure 3, there are five buds formed on the main branch. Three of them give branches of the second generation with three new buds on each of them. Branches of the second generation appear one after another when the main branch stops growing. It is interesting to note that one of the buds on the main branch gives 

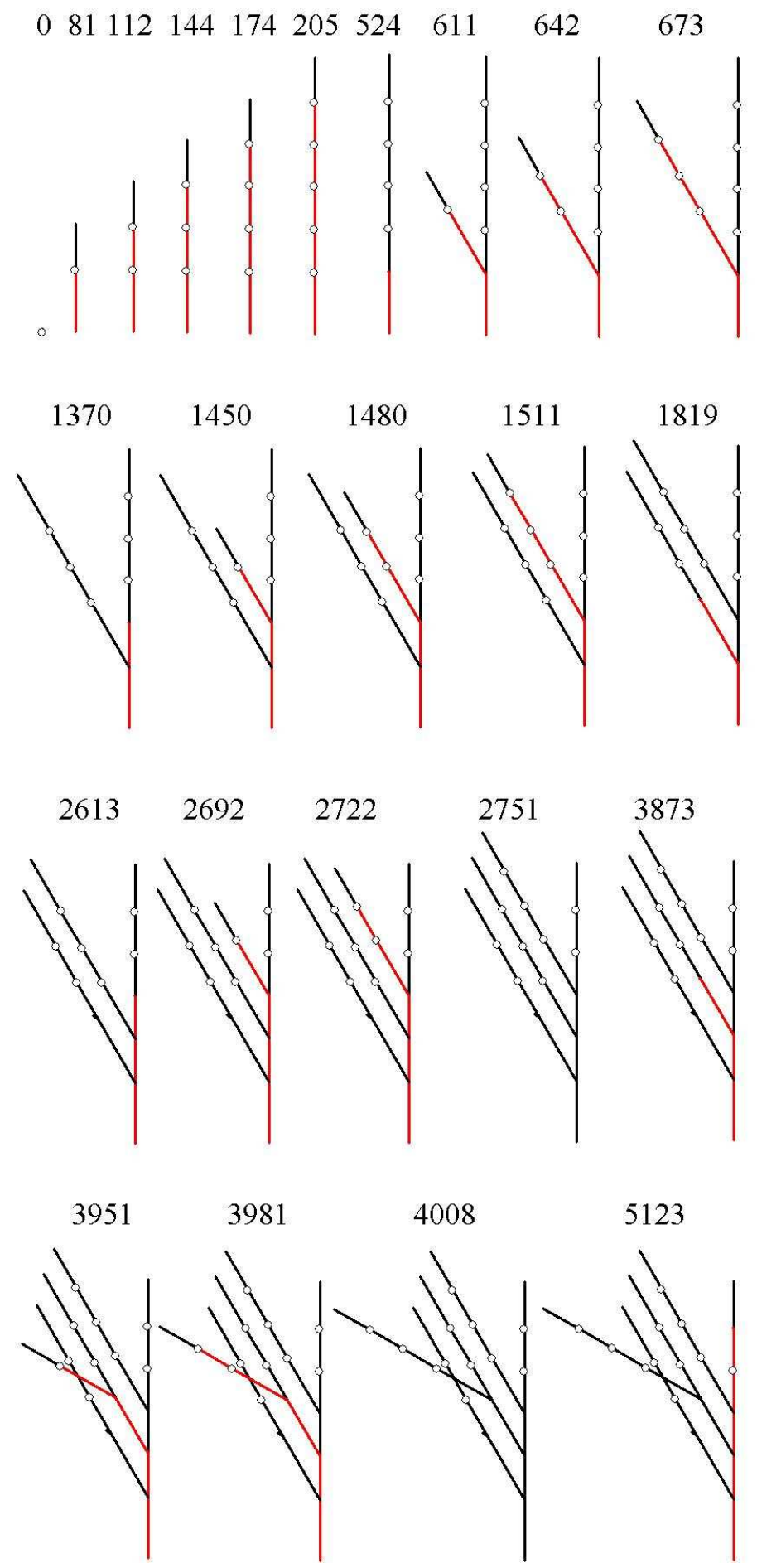

Figure 3: Consecutive stages of plant growth from a bud. First the main branch grows and new buds appear on it. When the main branch stops growing, these buds give new branches with some buds on it. Three generations of branches are shown. Red color shows the flux of nutrients. 
a rudimentary branch which stops growing right after it appears. There are also some branches of the third generation.

Other branching patterns and 2D simulations are shown in Figures 4 and 5 (see [3], [4] for more details).
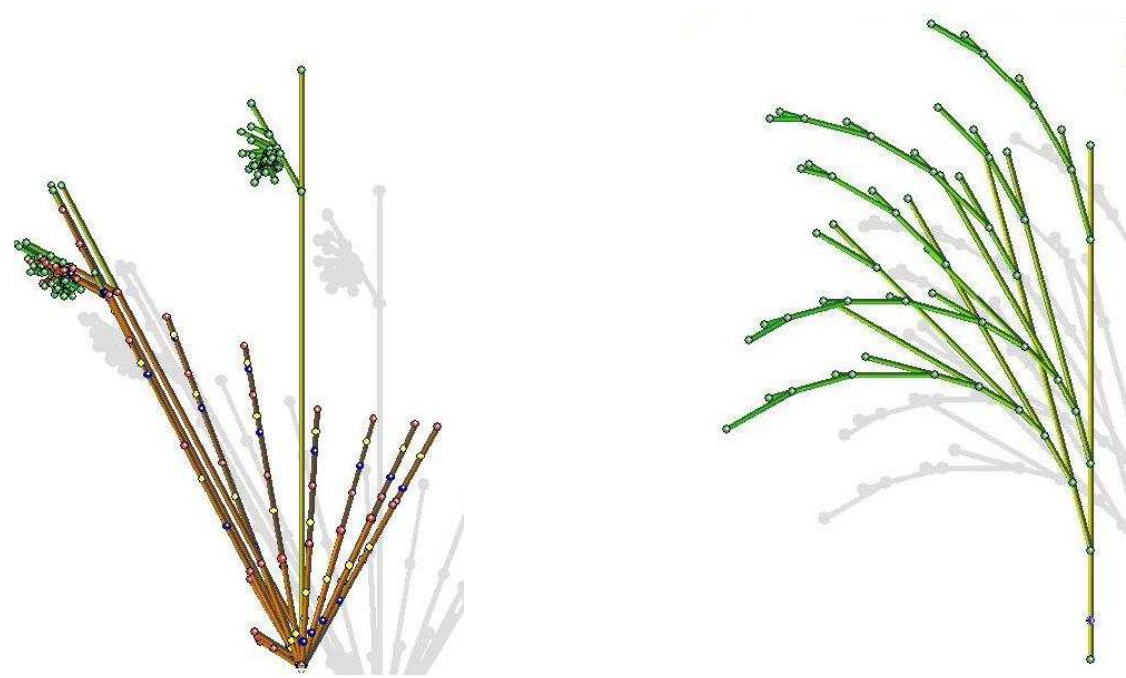

Figure 4: Possible branching patterns in the simulations with 1D model with branching.
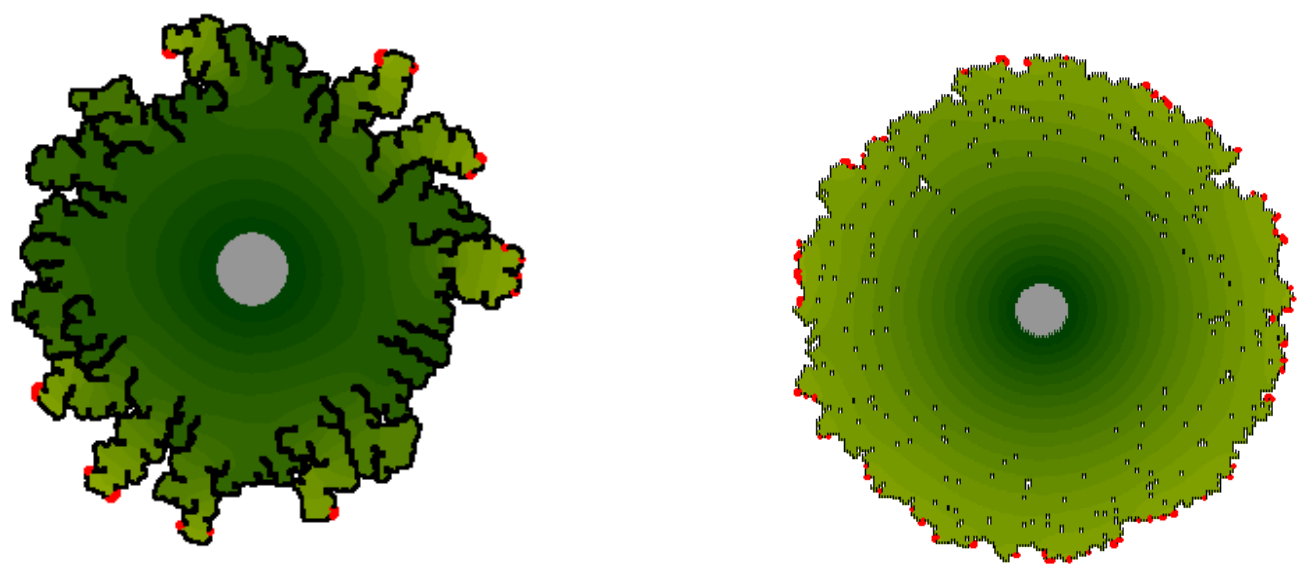

Figure 5: Characteristic patterns in 2D simulations. The model is presented in [3].

\section{Growth by basal meristem}

In this section we model growth of bulbous plants using the approach similar to that discussed above in the case of growth by apical meristem. The meristem in bulbous plants is located inside 
the bulb. Contrary to the apical meristem, it does not change its position during growth. Cell proliferation in the meristem provides growth of leaves. Nutrients come to the bulb from the roots. Thus, we can consider a simplified model where the meristem is represented as one-dimensional interval or a two-dimensional rectangle. The rate of cell proliferation in the meristem is determined by the concentrations of nutrients and of the GM-factor. We consider the following system of equations

$$
\begin{gathered}
\frac{\partial C}{\partial t}=d \Delta C+K\left(C_{0}-C\right)-[f(R)+g(R)] C, \\
\frac{\partial R}{\partial t}=\epsilon \Delta R+C g(R)-\sigma R .
\end{gathered}
$$

The first equation describes the concentration of nutrients $C$ in the meristem. Here the first term in the right-hand side describes their diffusion along the meristem, and the second term supply of nutrients from the roots. It is supposed to be proportional to the difference between some constant concentration of nutrients $C_{0}$ in the bulb or in the soil and the current concentration $C(x, t)$ which depends on space point and on time. We see here the first difference with the model of growth by apical meristem. Supply of nutrients in the latter is provided by convective flux along the stem (the second term in the left-hand side of (2.1)).

The last term in the right-hand side of (3.1) describes consumption of nutrients in the meristem. There are two mechanisms of consumption: nutrients are consumed in the GM-factor production and in leaf growth. Similar to the model considered in Section 2.1, the function $f(R)$ determines the proliferation rate of meristem cells. The difference is that in the previous model it corresponds to the stem growth, in the model considered here, this is growth of leaves,

$$
\frac{d L}{d t}=f(R) C
$$

where $L(x, t)$ is the length of the leaf at the point $x$ of the meristem.

Finally, we note that equation (3.2) is similar to equation (2.2) (with $F=1$ ). We consider that GM-factors can diffuse, yet their diffusion coefficient $\epsilon$ is supposed to be small compared to the diffusion coefficient of nutrients $d$. From the biological point of view, this term describes diffusion of the mitosis factor or, more general, stimulation of cell proliferation by neighboring cells. We are not aware whether this mechanism is observed in plant meristem. From the modelling point of view, however, it smoothes the system.

\subsection{Linear stability analysis}

The kinetic system

$$
\begin{aligned}
& \frac{d C}{d t}=F(C, R), \\
& \frac{d R}{d t}=G(C, R),
\end{aligned}
$$

where

$$
F(C, R)=K\left(C_{0}-C\right)-[f(R)+g(R)] C, \quad G(C, R)=C g(R)-\sigma R
$$


can have from one to three stationary points. Let us study stability of a stationary point $\left(C^{*}, R^{*}\right)$ with respect to the system

$$
\begin{aligned}
& \frac{\partial C}{\partial t}=d \Delta C+F(C, R), \\
& \frac{\partial R}{\partial t}=\epsilon \Delta R+G(C, R)
\end{aligned}
$$

in a rectangular domain $\Omega=\left\{0 \leq x \leq L_{x}, 0 \leq y \leq L_{y}\right\}$ with the homogeneous Neumann boundary conditions. Consider the spectrum of the problem linearized about this point:

$$
\begin{aligned}
& d \Delta C+F_{C}^{\prime}\left(C^{*}, R^{*}\right) C+F_{R}^{\prime}\left(C^{*}, R^{*}\right) R=\lambda C, \\
& \epsilon \Delta R+G_{C}^{\prime}\left(C^{*}, R^{*}\right) C+G_{R}^{\prime}\left(C^{*}, R^{*}\right) R=\lambda R .
\end{aligned}
$$

Suppose that the matrix

$$
J_{0}=\left(\begin{array}{ccc}
F_{C}^{\prime}\left(C^{*}, R^{*}\right) & , & F_{R}^{\prime}\left(C^{*}, R^{*}\right) \\
G_{C}^{\prime}\left(C^{*}, R^{*}\right) & , & G_{R}^{\prime}\left(C^{*}, R^{*}\right)
\end{array}\right)
$$

has both eigenvalues in the left-half plane. Denote by $\mu_{i}(\xi), i=1,2$ the eigenvalues of the matrix

$$
J(\xi)=\left(\begin{array}{ccc}
F_{C}^{\prime}\left(C^{*}, R^{*}\right)-d \xi^{2} & , & F_{R}^{\prime}\left(C^{*}, R^{*}\right) \\
G_{C}^{\prime}\left(C^{*}, R^{*}\right) & , & G_{R}^{\prime}\left(C^{*}, R^{*}\right)-\epsilon \xi^{2}
\end{array}\right) .
$$

Both of them have negative real parts for $\xi=0$ and for $|\xi|$ sufficiently large.

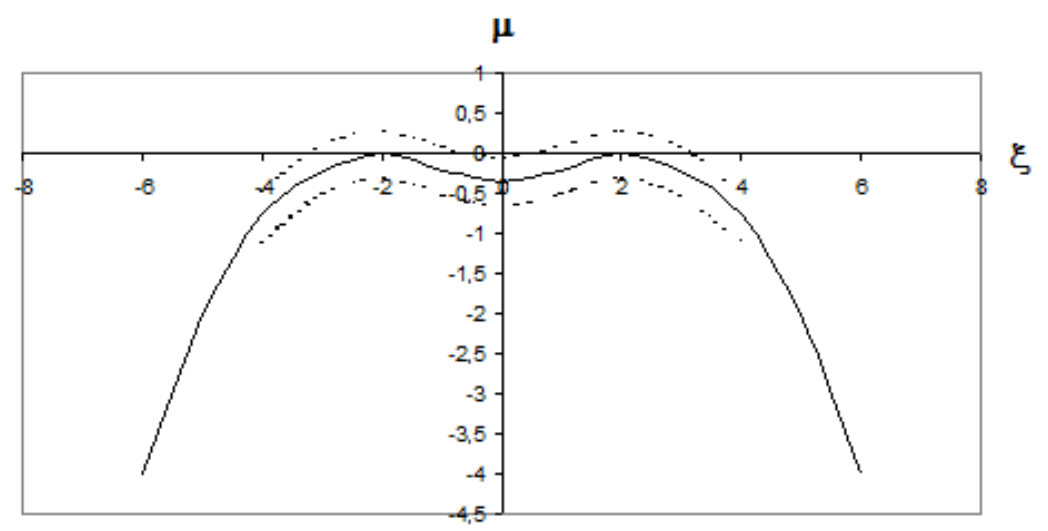

Figure 6: Schematic representation of the principal eigenvalue of the matrix $A(\xi)$ as a function of $\xi$. For a critical value of parameter, the function $\mu_{1}(\xi)$ is negative except for $\xi= \pm 2$ where it equals zero.

Suppose that for some values of parameters, the function $\mu_{1}(\xi)$ is negative for all $\xi$ except for some values $\xi= \pm \xi_{0}$ such that $\mu_{1}\left( \pm \xi_{0}\right)=0$. The second eigenvalue $\mu_{2}(\xi)$ is supposed to be negative for all $\xi$. Let $d$ be the bifurcation parameter and $d=d_{c}$ its value for which the function 
$\mu_{1}(\xi)$ has such behavior. To show the dependence of the eigenvalue on $d$, we will use the notation $\mu_{1}(\xi, d)$. Suppose that

$$
\mu_{1}(\xi, d) \gtrless \mu_{1}\left(\xi, d_{c}\right), \quad d \gtrless d_{c}
$$

for $d$ sufficiently close to $d_{c}$ and for $\xi$ sufficiently close to $\pm \xi_{0}$ (Figure 6). Then for $d>d_{c}$ the constant solution $\left(C^{*}, R^{*}\right)$ can lose its stability resulting in appearance of a periodic in space solution. These are so-called Turing or dissipative structures.

If we consider a $1 \mathrm{D}$ problem in the interval $[0, L]$, then its length should correspond to the periodicity of the bifurcating solution, $L=2 \pi / \xi_{0}$. If the length is different, then the value of $\xi$ and the critical value of the bifurcation parameter will also change. In the $2 \mathrm{D}$ case in rectangle, $\xi^{2}=k_{x}^{2}+k_{y}^{2}$, where $k_{x}$ and $k_{y}$ are the wavenumbers in the $x$ and $y$ directions. They are determined by the dimensions $L_{x}$ and $L_{y}$ of the rectangle.

Let focus on the particular case of functions $F$ and $G$ given by (3.6). Details of hereafter calculations are given in Appendix A.

The matrix $J_{0}$, defined in (3.11), has both eigenvalues with negative real parts if and only if (see Proposition 2 in Appendix A)

$$
K C_{0}+g\left(R^{*}\right) \alpha\left(R^{*}\right) \beta^{\prime}\left(R^{*}\right)>0 \quad \text { and } \quad\left(\frac{\beta}{\alpha}\right)^{\prime}\left(R^{*}\right)>0
$$

where functions $\alpha$ and $\beta$ are given by

$$
\alpha(R)=\frac{K C_{0}}{K+f(R)+g(R)} \quad \text { and } \quad \beta(R)=\frac{\sigma R}{g(R)} .
$$

Under this assumption, a Turing instability, as described above, can occur (see Proposition 3 in Appendix A) if $\beta^{\prime}\left(R^{*}\right)<0$ and $\epsilon$ is small. Indeed, there exists $d_{c}>0$ such that the stationary solution $\left(C^{*}, R^{*}\right)$ is linearly stable for $d<d_{c}$ and is unstable for $d>d_{c}$, with

$$
d_{c}=\frac{K C_{0} g\left(R^{*}\right)\left(\frac{\beta}{\alpha}\right)^{\prime}\left(R^{*}\right)+\epsilon \xi^{2}\left[K+f\left(R^{*}\right)+g\left(R^{*}\right)\right]}{\xi^{2}\left[-g\left(R^{*}\right) \beta^{\prime}\left(R^{*}\right)-\epsilon \xi^{2}\right]} .
$$

\subsection{Numerical simulations}

We begin numerical simulations with the 1D model of growth by basal meristem

$$
\begin{aligned}
\frac{\partial C}{\partial t} & =d \frac{\partial^{2} C}{\partial x^{2}}+K\left(C_{0}-C\right)-[f(R)+g(R)] C, \\
\frac{\partial R}{\partial t} & =\epsilon \frac{\partial^{2} R}{\partial x^{2}}+C g(R)-\sigma R .
\end{aligned}
$$

We recall that we will consider a small diffusion coefficient $\epsilon$, compared to $d$. 


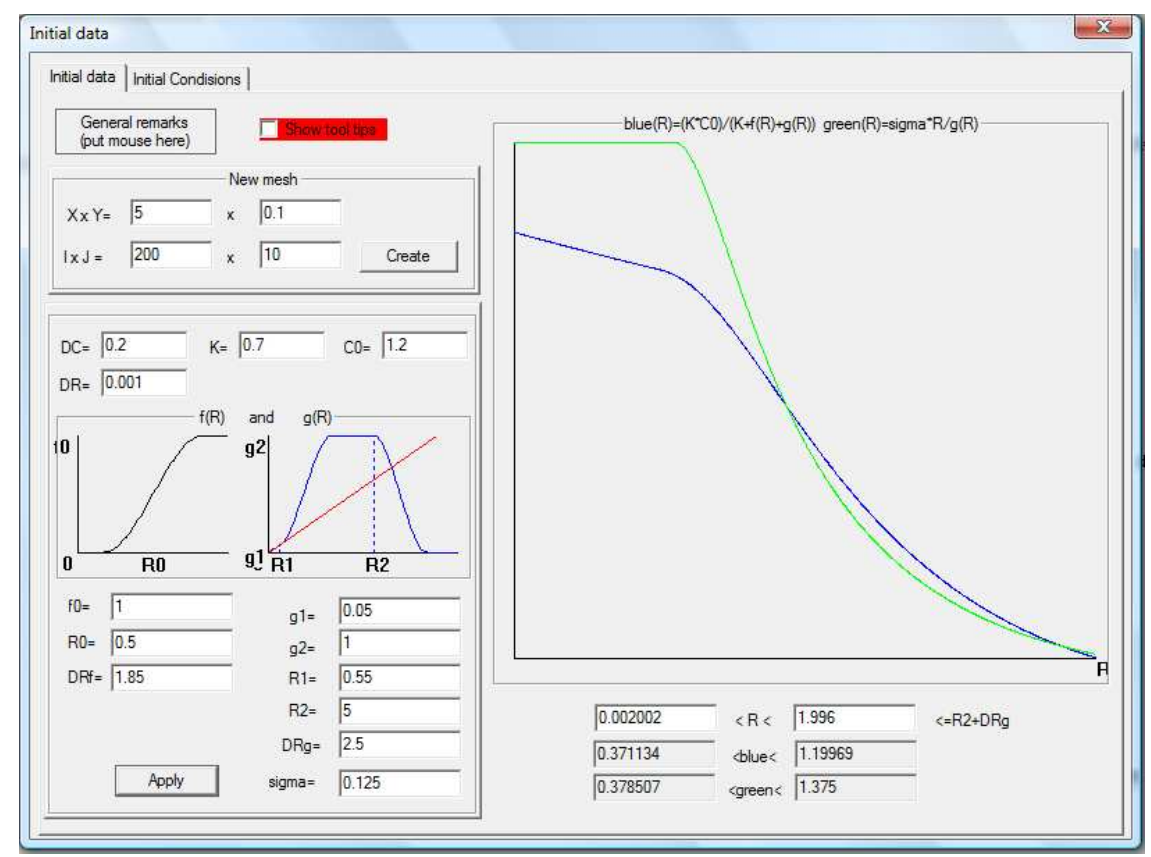

Figure 7: User interface with the values of parameters, the graphs of the functions $f$ and $g$, the functions $\alpha$ and $\beta$ (right panel).

Figure 7 shows the numerical interface used to compute the solutions of (3.12)-(3.13), with the values of the parameters, the form of the functions $f$ and $g$, which can be modified by the user, and the null lines of the ordinary differential system of equations (3.4)-(3.5) with (3.6) (right panel). For the given values of parameters, there are three stationary points:

$$
C=C_{0}=1.2, R=0 ; C=0.87, R=0.93 ; C=0.39, R=1.87 \text {. }
$$

The first one is stable both with respect to the kinetic system and with respect to the reactiondiffusion system (indeed, $\sigma-C_{0} g^{\prime}(0)=0.065>0$, see Proposition 1 ). The second one is unstable (the second condition in Proposition 2 is not satisfied). The last point is stable with respect to the kinetic system (see Proposition 2) but can lose its stability due to diffusion. In this case, Turing or dissipative structures can appear. We begin with investigation of such structures in the 1D case.

Figure 8 shows the bifurcation diagram where the diffusion coefficient $d$ in the first equation is chosen as bifurcation parameter. The solution with 4.5 periods is observed in a relatively narrow interval of values of $d$, from 0.082 to 0.42 . If we move along this branch of solutions, then for $d$ sufficiently large there is a transition to another branch with 3.5 periods. Then from this branch to the branch with 3 periods. For small values of $d$, we observe transition from the 3-period branch to the 4-period branch. This transition is shown in Figure 9.

It is interesting to note that lower critical values of $d$ for which the branches appear are approximately the same for all types of solutions. However, their bifurcations can be different. The numerical results allow us to suggest that it is a supercritical bifurcation for the 4.5-period branch. At the same time it can be a subcritical bifurcation for other branches (shown schematically as a 


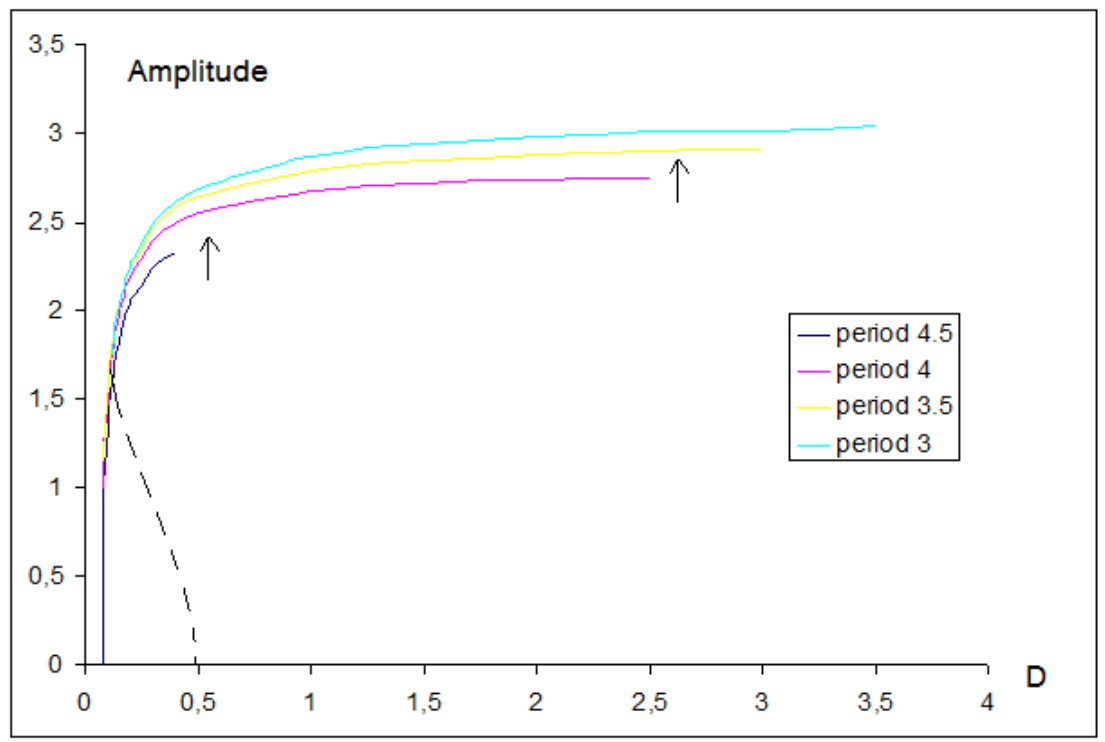

Figure 8: Bifurcation diagram in the 1D case. The amplitude of stationary solutions is defined as its maximum minus its minimum. Each curve corresponds to a solution with given periodicity.

dashed line in Figure 8). For example, if we move along the branch with 4-periodic solutions and decrease the value of $d$, then the amplitude of the solution converges to zero for $d=0.082$, it remains zero for $d=0.083$ and it growth for $d=0.084$ but in the form of 4.5-periodic solution. This suggests us to conclude that the branch of 4-periodic solutions bifurcates from zero subcritically for greater values of $d$.

Typical solutions in the 2D case are shown in Figures 10.
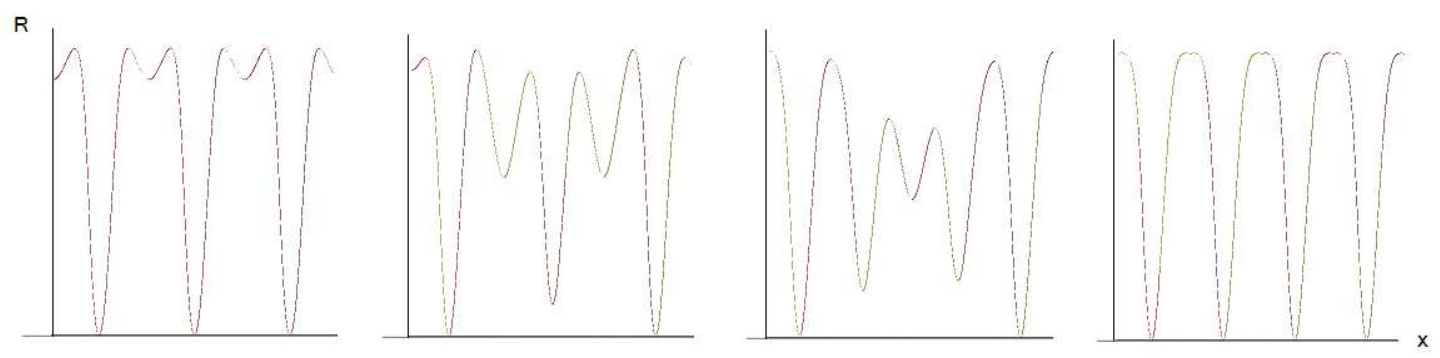

Figure 9: Distributions of $R$ in consecutive moments of time showing transition from a 3-periodic to a 4-periodic solution. 

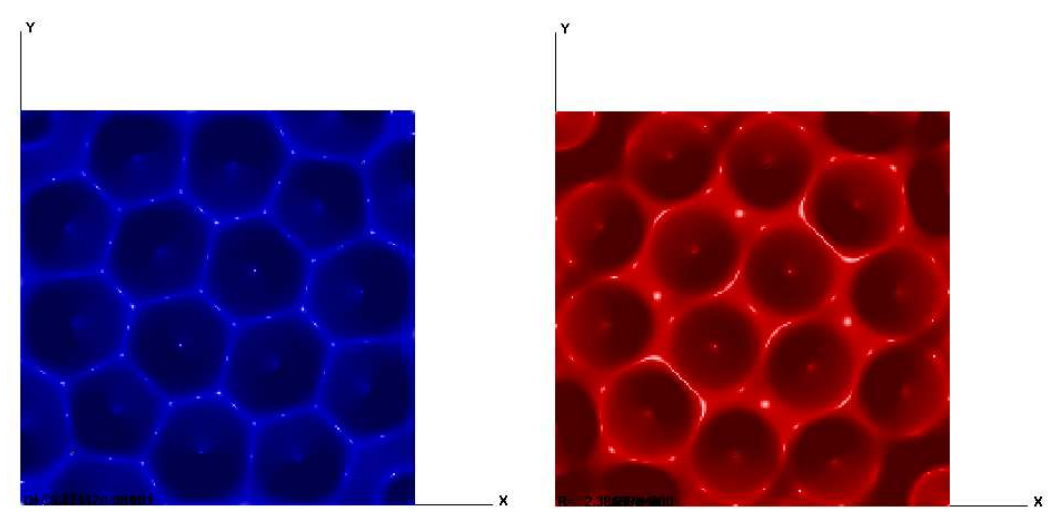

Figure 10: Distributions of $C$ (left) and $R$ (right) in the 2D case. Here $d=0.082$, all other values of parameters are the same as in the 1D case.

\section{Bifurcation of waves periodic at infinity}

Let us recall that system (3.12), (3.13) has three stationary points (3.14). Denote them by $P_{0}, P_{1}$ and $P_{2}$, respectively. For the kinetic system two of them $P_{0}$ and $P_{2}$ are stable, the intermediate point $P_{1}$ is unstable. If we take diffusion into account, the point $P_{0}$ remains stable, while $P_{2}$ can be stable or unstable. In the latter case, periodic in space structures emerge. We discussed them in the previous section.

Consider first the case where the point $P_{2}$ is stable. We can expect the existence of a travelling wave between the points $P_{0}$ and $P_{2}$. This is a solution of system (3.12), (3.13) of the form $C(x, t)=$ $c(x-\nu t), R(x, t)=r(x-\nu t)$, where $\nu$ is the wave speed. Existence of such waves is confirmed by numerical simulations (Figure 11). The wave propagates from the right to the left. The values of $C$ and $R$ corresponding to the stationary point $P_{0}$ are in front of the transition zone, the values corresponding to the points $P_{2}$ behind it.

The wave is a stationary solution of the system

$$
\begin{aligned}
& \frac{\partial C}{\partial t}=d \frac{\partial^{2} C}{\partial x^{2}}+\nu \frac{\partial C}{\partial x}+F(C, R), \\
& \frac{\partial R}{\partial t}=\epsilon \frac{\partial^{2} R}{\partial x^{2}}+\nu \frac{\partial R}{\partial x}+G(C, R),
\end{aligned}
$$

where, as above,

$$
F(C, R)=K\left(C_{0}-C\right)-[f(R)+g(R)] C, \quad G(C, R)=C g(R)-\sigma R .
$$

In order to discuss the wave stability, let us consider the spectrum of the problem linearized about the wave:

$$
\begin{aligned}
d C^{\prime \prime}+\nu C^{\prime}+F_{C}^{\prime}(c(x), r(x)) C+F_{R}^{\prime}(c(x), r(x)) R & =\lambda C \\
\epsilon R^{\prime \prime}+\nu R^{\prime}+G_{C}^{\prime}(c(x), r(x)) C+G_{R}^{\prime}(c(x), r(x)) R & =\lambda R .
\end{aligned}
$$




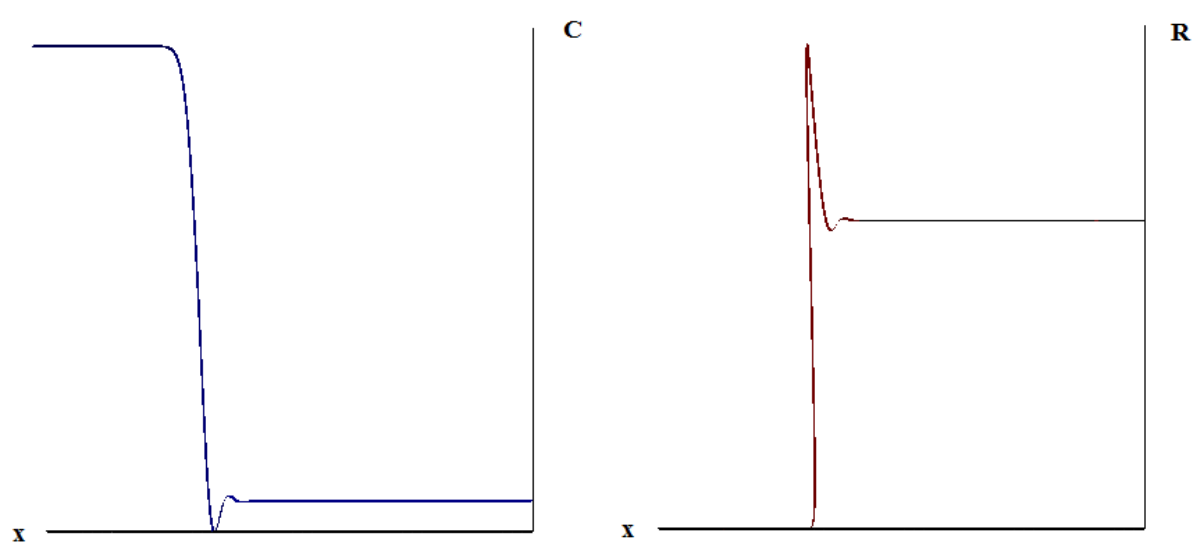

Figure 11: Propagation of travelling wave. Snapshot of the spatial distributions of $C$ (left) and $R$ (right).

It can be easily verified that the derivative $\left(c^{\prime}(x), r^{\prime}(x)\right)$ of the wave is the eigenfunction corresponding to the zero eigenvalue. If all other spectrum of the linearized system lies in the left half-plane, then the wave is asymptotically stable with shift [24]. If a simple real eigenvalue crosses the origin, then the wave loses its stability and some other stationary waves will bifurcate. Another possible situation is when a pair of complex conjugate eigenvalues crosses the imaginary axis. Then a Hopf like bifurcation occurs resulting in appearance of periodic in time solutions. These bifurcations of travelling waves are well known and intensively studied in relation with flame propagation and with numerous other applications [24].

We will discuss here another situation where a part of the essential spectrum crosses the imaginary axis. In order to determine the essential spectrum of this system, denote by $\left(c_{0}, r_{0}\right)$ the limits of the functions $c(x)$ and $r(x)$ at plus infinity (behind the wave front) and consider the spectral problem

$$
\begin{aligned}
& d C^{\prime \prime}+\nu C^{\prime}+F_{C}^{\prime}\left(c_{0}, r_{0}\right) C+F_{R}^{\prime}\left(c_{0}, r_{0}\right) R=\lambda C, \\
& \epsilon R^{\prime \prime}+\nu R^{\prime}+G_{C}^{\prime}\left(c_{0}, r_{0}\right) C+G_{R}^{\prime}\left(c_{0}, r_{0}\right) R=\lambda R .
\end{aligned}
$$

We introduce the matrix

$$
B(\xi)=\left(\begin{array}{ccc}
F_{C}^{\prime}\left(c_{0}, r_{0}\right)-d \xi^{2}+\nu i \xi & F_{R}^{\prime}\left(c_{0}, r_{0}\right) \\
G_{C}^{\prime}\left(c_{0}, r_{0}\right) & , & G_{R}^{\prime}\left(c_{0}, r_{0}\right)-\epsilon \xi^{2}+\nu i \xi
\end{array}\right)
$$

and denote by $\lambda_{j}(\xi), j=1,2$ its eigenvalues. By definition, the essential spectrum of the linear system in the left-hand side of (4.3), (4.4) is the set of all complex $\lambda_{1}(\xi)$ and $\lambda_{2}(\xi)$ for all $\xi \in \mathbb{R}$. In fact, it is only the part of the essential spectrum, which corresponds to plus infinity. A similar construction should be done at minus infinity.

The eigenvalues of the matrix $B(\xi)$ can be expressed through the eigenvalues of the matrix $A(\xi)$ (Section 3):

$$
\lambda_{j}(\xi)=\mu_{j}(\xi)+\nu i \xi, \quad j=1,2 .
$$


The curve $\lambda_{1}(\xi)$ for the critical values of the parameters is schematically shown in Figure 12 (cf. Figure 6). It touches the imaginary axis at two points, $\lambda\left(\xi_{0}\right)= \pm i \nu \xi_{0}$. For the value of the parameter exceeding the critical one, the essential spectrum is partially in the right-half plane. Therefore, the wave $\left(c_{0}(x), r_{0}(x)\right)$ can lose its stability. However, the usual bifurcation analysis is not applicable here since these are not isolated eigenvalues.

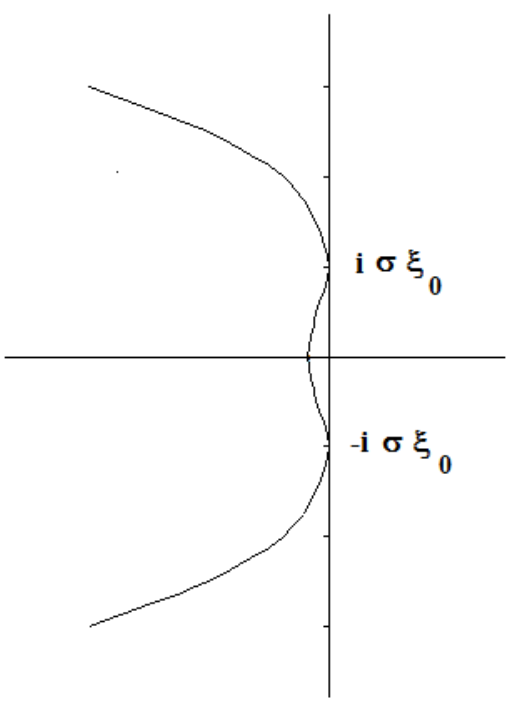

Figure 12: Schematic representation of the essential spectrum for the critical values of the parameters.

Thus, emergence of Turing structures around the stationary point $P_{2}$ moves the essential spectrum to the right-half plane. This can result in a possible instability of the wave. An example of numerical simulations is shown in Figure 13. The wave propagates to the left, and a periodic spatial structure emerges behind it. Moreover, this structure propagates also to the right. Therefore, there is another periodic wave which provides transition between an unstable point $P_{2}$ and a Turing structure. Another effect is shown in Figure 14. There is a transition from a 1D periodic solution to a $2 \mathrm{D}$ periodic solution.

\section{Conclusions}

Plant growth is determined by fluxes of nutrients and metabolites and by cell proliferation in the meristem. An important feature of plants is that cell proliferation is strongly localized in space. In the case of apical meristem, plant growth can be described mathematically as a free boundary problem where motion of the boundary occurs due to cell proliferation. In the case of basal meristem, it does not change its position. Cell proliferation in the meristem results in leaf growth.

Plant phyllotaxis is not yet well understood. From the mathematical point of view, it is related 


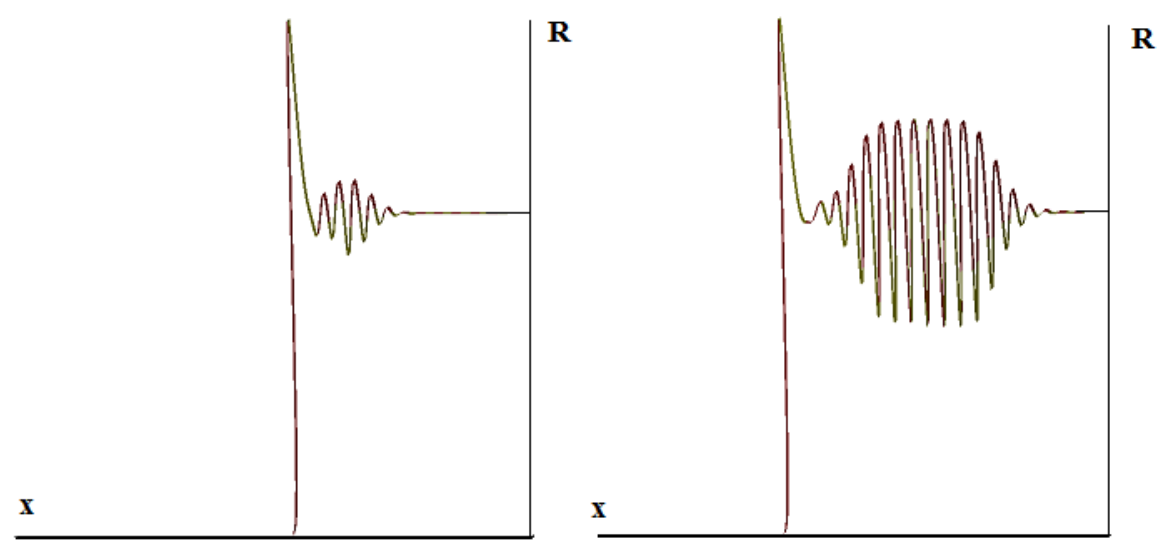

Figure 13: Turing structure emerges behind the wave. The periodic solution spreads in space. Thus, there are two waves. One moves to the left and provides transition between the point $P_{0}$ and the periodic solution, another one moves to the right and provides transition between the point $P_{2}$ and the periodic solution.
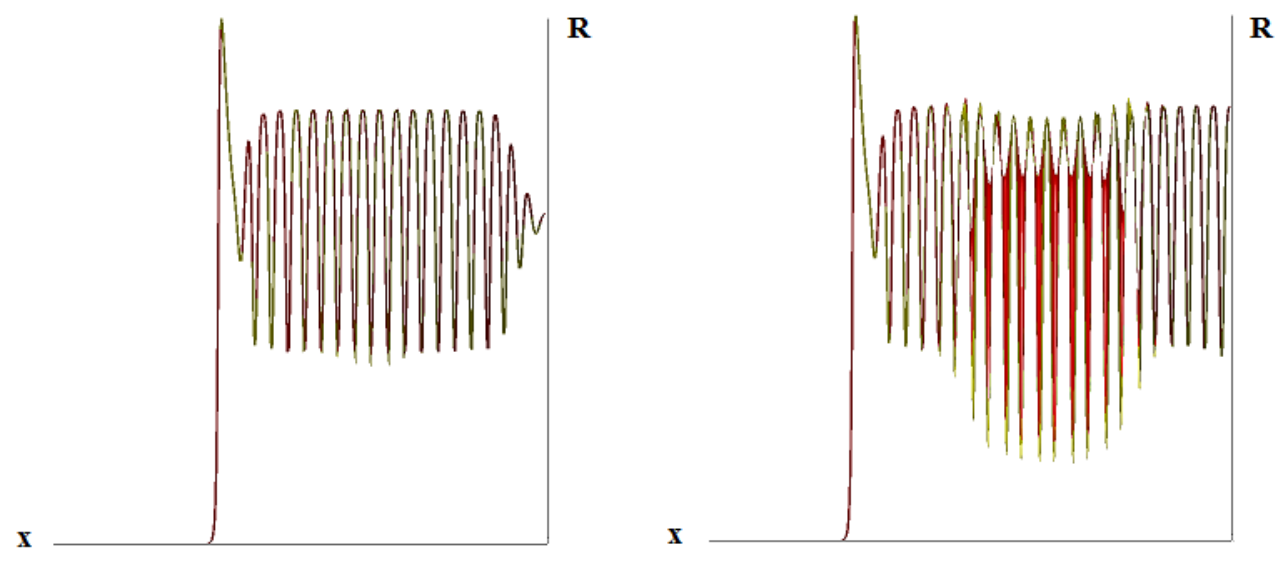

Figure 14: 1D periodic in space solution behind the wave is unstable. A 2D solution emerges and propagates in both directions. 
to nonlinear dynamics and pattern formation. There are various possible mechanisms of pattern formation in plants. Some of them were discussed in this paper.

There are some interesting aspects of nonlinear dynamics in plants related to biological diversity. It is important to note that the models considered in this work possess continuous families of stationary solutions. In the 1D case it can be all intervals with length greater than the minimal one. In the 2D case it can be practically all plane forms with some nonrestrictive conditions on the curvature [3]. The differences in the initial conditions and external conditions result in differences in solutions or, in the other words, among individuals of the same species. This is possibly due to the fact that all solutions are possible.

What happens if we change the parameters of the model? Its second fundamental property is that stationary solutions exist for all physically realistic values of parameters. Therefore, not only the individuals of a given species can have an arbitrary form but individuals of any species can have any possible form. This is why there are so many different forms and different species. Of course, this is only a theoretical possibility. The existence of stationary solutions does not necessary mean that the solution of the evolution problem with some specific initial condition will converge to them.

If we slightly change the parameters of the model, the initial condition or the external conditions (that is the boundary conditions in the model), or the space and time steps in numerical simulations, we may not obtain the convergence to exactly the same stationary solutions. We can expect that if the difference in parameters is small, the solutions will be close to each other. It appears, however, that this is not necessarily so. In some cases, the solutions are close to each other, while in some other cases they can be essentially different. The former case corresponds to individuals of the same species, the latter, to transition to other species.

We come now to the question about the structural stability of solutions: if the values of parameters approach each other, will the solutions also converge to each other? The results of the numerical simulations show that this is probably not the case. When we decrease the time and space steps we do not obtain the convergence of solutions. At first glance this contradicts the unwritten convention of numerical modelling: solutions must converge. However, there is no contradiction. The usual expectation that solutions converge concerns a fixed and finite time interval. This rule is not applicable to the longtime behavior. What does longtime behavior mean? Of course, numerical simulations are always carried out on a finite time interval. To explain this, consider a typical situation in the theory of dynamical systems in which a stationary solution can have a stable manifold along which solutions of the evolution problem approach the stationary solution as time increases, and unstable manifold along which solutions of the evolution problem diverge from the stationary solution. If the initial condition is close to the stable manifold but it is not located exactly on it, then in the beginning the solution of the evolution problem will approach the stationary solution, but after some time diverge from it. For two different but close initial conditions, the solutions of the evolution problem will first approach each other, but then at the second stage the difference between them will increase and after some time they can become completely different.

How is this description related to the modelling of plant growth? The solution of the evolution problem approaches some unstable stationary solution along its stable manifolds and then moves away from it along the unstable manifold (see [3] for more detail). Small perturbations grow 
exponentially, and convergence of solutions, when we decrease the time and space steps, does not take place.

Thus, solutions are not structurally stable in the usual sense. However, they can be structurally stable in some averaged sense: changing the parameters, we can observe similar structures with the same characteristic dimensions, though different in small details. The conclusion that biological forms can be structurally stable in some averaged sense is in agreement with the fact that individuals from the same species, though different from each other, still have many features in common. On the other hand, in some cases structural stability does not take place even in average. The presence of stable and unstable manifolds can result in essential difference of solutions with close initial conditions. This situation can be considered as transition to new biological species.

\section{Acknowledgements}

We started this work together with Loïc Forest. A tragic accident took him away. We dedicate this paper to the memory of this bright researcher and nice person. His work on auxin transport in plants [8] is an important contribution to modelling of plant growth.

This work was partially supported by the Rhone-Alpes Institute of Complex Systems (IXXI).

\section{A Appendix: Linear Stability Analysis}

We develop in this section the stability analysis of the reaction-diffusion system

$$
\begin{aligned}
& \frac{\partial C}{\partial t}=d \Delta C+K\left(C_{0}-C\right)-[f(R)+g(R)] C, \\
& \frac{\partial R}{\partial t}=\epsilon \Delta R+C g(R)-\sigma R,
\end{aligned}
$$

where $f$ and $g$ are nonnegative differentiable functions, with $f(0)=g(0)=0$, and $f$ is nondecreasing (see Figure 7).

A stationnary solution $(\bar{C}, \bar{R})$ of (A1)-(A2) satisfies

$$
\begin{aligned}
{[K+f(\bar{R})+g(\bar{R})] \bar{C} } & =K C_{0} \\
\sigma \bar{R} & =\bar{C} g(\bar{R}) .
\end{aligned}
$$

It is then obvious that $(\bar{C}, \bar{R})=\left(C_{0}, 0\right)$ is a stationnary solution of (A1)-(A2). Non-trivial homogeneous stationnary solutions $\left(C^{*}, R^{*}\right) \neq\left(C_{0}, 0\right)$ verify

$$
C^{*}=\frac{K C_{0}}{K+f\left(R^{*}\right)+g\left(R^{*}\right)} \quad \text { and } \quad C^{*}=\frac{\sigma R^{*}}{g\left(R^{*}\right)} .
$$

We define, for $R>0$,

$$
\alpha(R)=\frac{K C_{0}}{K+f(R)+g(R)}
$$


and

$$
\beta(R)=\frac{\sigma R}{g(R)} .
$$

Then, from (A3), a homogeneous stationary solution $\left(C^{*}, R^{*}\right)$ of (A1)-(A2) satisfies

$$
\alpha\left(R^{*}\right)=\beta\left(R^{*}\right) \quad \text { and } \quad C^{*}=\alpha\left(R^{*}\right) .
$$

\section{A1. Linearization and Characteristic Equation}

The linearization of system (A1)-(A2) about $\left(C^{*}, R^{*}\right)$ leads to

$$
\begin{aligned}
& \frac{\partial C}{\partial t}=d \Delta C-\left[K+f\left(R^{*}\right)+g\left(R^{*}\right)\right] C-C^{*}\left[f^{\prime}\left(R^{*}\right)+g^{\prime}\left(R^{*}\right)\right] R, \\
& \frac{\partial R}{\partial t}=\epsilon \Delta R+g\left(R^{*}\right) C-\left[\sigma-C^{*} g^{\prime}\left(R^{*}\right)\right] R .
\end{aligned}
$$

From (A3), that is for $R^{*} \neq 0$, we get

$$
\sigma-C^{*} g^{\prime}\left(R^{*}\right)=g\left(R^{*}\right) \beta^{\prime}\left(R^{*}\right) .
$$

Looking for solutions of (A6)-(A7) in the form

$$
C(x, t)=c(t) e^{i \xi x}, \quad R(x, t)=r(t) e^{i \xi x}, \quad k \geq 0,
$$

one finds that

$$
\frac{d}{d t}\left(\begin{array}{c}
c(t) \\
r(t)
\end{array}\right)=J\left(\begin{array}{c}
c(t) \\
r(t)
\end{array}\right)
$$

where

$$
J=\left(\begin{array}{cc}
-\left[K+f\left(R^{*}\right)+g\left(R^{*}\right)\right]-\xi^{2} d & -C^{*}\left[f^{\prime}\left(R^{*}\right)+g^{\prime}\left(R^{*}\right)\right] \\
g\left(R^{*}\right) & -g\left(R^{*}\right) \beta^{\prime}\left(R^{*}\right)-\epsilon \xi^{2}
\end{array}\right) .
$$

Since it will be helpful in the following, let us define

$$
J_{0}=\left(\begin{array}{cc}
-\left[K+f\left(R^{*}\right)+g\left(R^{*}\right)\right] & -C^{*}\left[f^{\prime}\left(R^{*}\right)+g^{\prime}\left(R^{*}\right)\right] \\
g\left(R^{*}\right) & -g\left(R^{*}\right) \beta^{\prime}\left(R^{*}\right)
\end{array}\right) .
$$

The linear stability of $\left(C^{*}, R^{*}\right)$ is related to the signs of $\operatorname{tr}(J)$ and $\operatorname{det}(J)$, where

$$
\begin{aligned}
\operatorname{tr}(J) & =\operatorname{tr}\left(J_{0}\right)-(d+\epsilon) \xi^{2}, \\
\operatorname{det}(J) & =\operatorname{det}\left(J_{0}\right)+\left[d g\left(R^{*}\right) \beta^{\prime}\left(R^{*}\right)+\epsilon\left(K+f\left(R^{*}\right)+g\left(R^{*}\right)\right] \xi^{2}+\epsilon d \xi^{4} .\right.
\end{aligned}
$$

Indeed, $\left(C^{*}, R^{*}\right)$ is linearly stable if and only if $\operatorname{tr}(J)<0$ and $\operatorname{det}(J)>0$. 


\section{A2. The case of the equilibrium $\left(C^{*}, R^{*}\right)=\left(C_{0}, 0\right)$}

The linearization of system (A1)-(A2) about $\left(C^{*}, R^{*}\right)=\left(C_{0}, 0\right)$ leads to the jacobian matrix

$$
J=\left(\begin{array}{cc}
-K-d \xi^{2} & -C_{0}\left[f^{\prime}(0)+g^{\prime}(0)\right] \\
0 & -\left[\sigma-C_{0} g^{\prime}(0)\right]-\epsilon \xi^{2}
\end{array}\right) .
$$

It follows that $\operatorname{tr}(J)<0$ if and only if $K+(d+\epsilon) \xi^{2}>C_{0} g^{\prime}(0)-\sigma$, and $\operatorname{det}(J)>0$ if and only if $\epsilon \xi^{2}>C_{0} g^{\prime}(0)-\sigma$. It is then obvious that $\operatorname{tr}(J)<0$ if $\operatorname{det}(J)>0$. We conclude to the stability of the solution $\left(C_{0}, 0\right)$ in the next proposition.

Proposition 1. The stationary solution $\left(C^{*}, R^{*}\right)=\left(C_{0}, 0\right)$ is linearly stable if and only if

$$
\epsilon \xi^{2}>C_{0} g^{\prime}(0)-\sigma
$$

In particular, it is stable if $\sigma>C_{0} g^{\prime}(0)$.

\section{A3. Stability without diffusion}

In the absence of diffusion, the jacobian matrix $J$ in (A8) reduces to $J_{0}$ in (A9). The stationary solution $\left(C^{*}, R^{*}\right)$ is linearly stable if and only if $\operatorname{tr}\left(J_{0}\right)<0$ and $\operatorname{det}\left(J_{0}\right)>0$. Using the definition of $\alpha$, in (A4), one can write

$$
\operatorname{tr}\left(J_{0}\right)=-\frac{K C_{0}+g\left(R^{*}\right) \alpha\left(R^{*}\right) \beta^{\prime}\left(R^{*}\right)}{\alpha\left(R^{*}\right)} .
$$

Moreover, with the definition of $\beta$ in (A5), easy computations lead to

$$
\operatorname{det}\left(J_{0}\right)=K C_{0} g\left(R^{*}\right)\left(\frac{\beta}{\alpha}\right)^{\prime}\left(R^{*}\right) .
$$

Then the following result is straightforward.

Proposition 2. The stationary solution $\left(C^{*}, R^{*}\right)$ is linearly stable without diffusion if and only if

$$
K C_{0}+g\left(R^{*}\right) \alpha\left(R^{*}\right) \beta^{\prime}\left(R^{*}\right)>0 \quad \text { and } \quad\left(\frac{\beta}{\alpha}\right)^{\prime}\left(R^{*}\right)>0 .
$$

\section{A4. The case of a positive equilibrium $\left(C^{*}, R^{*}\right) \neq\left(C_{0}, 0\right)$}

We now focus on the stability of $\left(C^{*}, R^{*}\right) \neq\left(C_{0}, 0\right)$, in the presence of diffusion. We will show, in particular, that the stationary solution, stable in the absence of diffusion, can become unstable due to diffusion.

One can first notice that $\beta^{\prime}\left(R^{*}\right) \geq 0$ implies that $\operatorname{tr}\left(J_{0}\right)<0$, and consequently, from (A10), that $\operatorname{tr}(J)<0$. Moreover, one also gets, from (A11), $\operatorname{det}(J)>0$. Consequently, $\left(C^{*}, R^{*}\right)$ is unconditionally linearly stable when $\beta^{\prime}\left(R^{*}\right) \geq 0$. 
Let assume that $\beta^{\prime}\left(R^{*}\right)<0$, and that the stationary solution is stable in the absence of diffusion, that is $\operatorname{tr}\left(J_{0}\right)<0$ and $\operatorname{det}\left(J_{0}\right)>0$. From (A10), one obtains $\operatorname{tr}(J)<0$.

We also assume in the following that $\epsilon$ is small. This implies, in particular, that

$$
g\left(R^{*}\right) \beta^{\prime}\left(R^{*}\right)+\epsilon \xi^{2}<0, \text { for large } \xi .
$$

From (A11), we obtain

$$
\operatorname{det}(J)>0 \quad \text { if and only if } \quad d<d_{c}
$$

where

$$
d_{c}=\frac{\operatorname{det}\left(J_{0}\right)+\epsilon \xi^{2}\left[K+f\left(R^{*}\right)+g\left(R^{*}\right)\right]}{\xi^{2}\left[-g\left(R^{*}\right) \beta^{\prime}\left(R^{*}\right)-\epsilon \xi^{2}\right]} .
$$

Hence, the stationary solution becomes unstable for $d>d_{c}$.

Proposition 3. If $\beta^{\prime}\left(R^{*}\right) \geq 0$, then the non-trivial stationary solution $\left(C^{*}, R^{*}\right)$ is unconditionally linearly stable.

Assume $\beta^{\prime}\left(R^{*}\right)<0$. If

$$
K C_{0}+g\left(R^{*}\right) \alpha\left(R^{*}\right) \beta^{\prime}\left(R^{*}\right)>0 \quad \text { and } \quad\left(\frac{\beta}{\alpha}\right)^{\prime}\left(R^{*}\right)>0
$$

and $\epsilon$ is small, then a Turing instability appears, there exists $d_{c}>0$ such that the stationary solution $\left(C^{*}, R^{*}\right)$ is linearly stable for $d<d_{c}$ and is unstable for $d>d_{c}$.

If $\left(C^{*}, R^{*}\right)$ is unstable in the absence of diffusion, with $\operatorname{tr}\left(J_{0}\right)>0$, then increasing the diffusion (through $d$ or $\epsilon$ ) will have a tendency to stabilize $\left(C^{*}, R^{*}\right)$, as expected.

If $\left(C^{*}, R^{*}\right)$ is unstable in the absence of diffusion, with $\operatorname{det}\left(J_{0}\right)<0$, however, an increase of the diffusion will not a priori stabilize the stationary solution. For instance, when $\epsilon$ is small and tends to zero, then

$$
\operatorname{det}(J)=\operatorname{det}\left(J_{0}\right)+g\left(R^{*}\right) \beta^{\prime}\left(R^{*}\right) d \xi^{2}<0, \quad \text { for all } d \geq 0 .
$$

Hence the stationary solution is always unstable in this case. It appears that only an increase of $\epsilon$ can stabilize $\left(C^{*}, R^{*}\right)$. Indeed, $\left(C^{*}, R^{*}\right)$ becomes stable for

$$
\epsilon \xi^{2}>-\frac{\operatorname{det}\left(J_{0}\right)+g\left(R^{*}\right) \beta^{\prime}\left(R^{*}\right) d \xi^{2}}{K+f\left(R^{*}\right)+g\left(R^{*}\right)+d \xi^{2}} .
$$

\section{References}

[1] B. Alberts, D. Bray, J. Lewis, M. Raff, K. Roberts, J.D. Watson. Biologie moléculaire de la cellule, 3th edn. Médecine-Sciences, Flammarion, 1995.

[2] N. Bessonov, V. Volpert. On a problem of plant growth. In: Patterns and waves. A. Abramian, S. Vakulenko, V. Volpert, Eds. St. Petersburg, 2003, pp. 323-337. 
[3] N. Bessonov, V. Volpert. Dynamic models of plant growth. Publibook, Paris, 2006.

[4] N. Bessonov, N. Morozova, V. Volpert. Modelling of branching patterns in plants. Bull. Math. Biology, 70 (2008), no. 3, 868-893.

[5] E. Boucheron, A. Guivarch, A. Azmi, W. Dewitte, H. Van Onckelen, D. Chriqui. Competency of Nicotiana tabacum L. stem tissues to dedifferentiate is associated with differential levels of cell cycle gene expression and endogenous cytokinins. Planta, 215 (2002), 267-278.

[6] V. Brukhin, N. Morozova. Plant growth and development - basic knowledge and current views. Math. Model. Nat. Phenom. Vol. 6, No. 2, 2011, pp. 1-53.

[7] DArcy Thompson. On growth and forms. The complete revised edition. Dover, New York, 1992.

[8] L. Forest, J. Demongeot. Cellular modelling of secondary radial growth in conifer trees: application to Pinus radiata (D Don). Bull. Math. Biol. 68 (2006), 753-784.

[9] K. Himanen, E. Boucheron, S. Vanneste, J. de Almeida Engler, D. Inze, T. Beeckman. Auxin mediated cell cycle activation during early lateral root initiation. Plant Cell, 14 (2002), 23392351.

[10] I. Hoffmann, P.R. Clarke, M.J. Marcote, E. Karsenti, G. Draetta. Phosphorylation and activation of human cdc25-C by cdc2-cyclin $\mathrm{B}$ and its involvement in the self-amplification of MPF at mitosis. EMBO J. 12 (1993), no. 1, 53-63.

[11] R. V. Jean. Phyllotaxis. A systematic study in plant morphogenesis. Cambridge University Press, New York, 1994.

[12] H. Jonsson, M.G. Heisler, B.E. Shapiro, E.M. Meyerowitz, E. Mjolsness. An auxin-driven polarized transport model for phyllotaxis. PNAS 103 (2006), no. 5, 1633-1638.

[13] N. Khiripet, R. Viruchpintu, J. Maneewattanapluk, J. Spangenberg, J. R. Jungck. Morphospace: measurement, modeling, mathematics, and meaning. Math. Model. Nat. Phenom., 6 (2011), No. 2, 54-81.

[14] Z. Magyar, L. De Veylder, A. Atanassova, L. Bako, D. Inze, L. Bogre. The role of the Arabidopsis E2FB transcription factor in regulating auxin-dependent cell division. Plant Cell, 17 (2005) no. 9, 2527-2541.

[15] H. Meinhardt, A.J. Koch, G. Bernasconi. Models of pattern formation applied to plant development. In: Symmetry in Plants, (D. Barabe and R. V. Jean, Eds), World Scientific Publishing, Singapore, 1998, 723-758.

[16] H. G. Othmer, K. Painter, D. Umulis, C. Xue. The intersection of theory and application in elucidating pattern formation in developmental biology. Math. Model. Nat. Phenom., 4 (2009), No. 4, 3-82. 
[17] D. Reinhardt, E.R. Pesce, P. Stieger, T. Mandel, K. Baltensperger, M. Bennett, J. Traas, J. Friml, C. Kuhlemeier. Regulation of phyllotaxis by polar auxin transport. Nature 462 (2003), 255-260.

[18] I.V. Rudskiy, G.E. Titova, T.B. Batygina. Analysis of space-temporal symmetry in the early embryogenesis of Calla palustris L., Araceae. Math. Model. Nat. Phenom., 6 (2011), No. 2, 82-106.

[19] F. Skoog, C.O. Miller. Chemical regulation of growth and organ formation in plant tissues cultured in vitro. Symp. Soc. Exp. Biol., 11 (1957), 118-140.

[20] R.S. Smith, S. Guyomarch, T. Mandel, D. Reinhardt, C. Kuhlemeier, P. Prusinkiewicz. A plausible model of phyllotaxis. PNAS, 103 (2006), no. 5, 1301-1306.

[21] R. Soni, J.P. Carmichael, Z.H. Shah, J.A. Murray. A family of cyclin D homologs from plants differentially controlled by growth regulators and containing the conserved retinoblastoma protein interaction motif. Plant Cell, 7 (1995) no. 1, 85-103.

[22] J. Traas, I. Bohn-Courseau. Cell proliferation patterns at the shoot apical meristem. Curr. Opin. Plant Biol. 8 (2005), 587-592.

[23] B.S. Treml, S. Winderl, R. Radykewicz, M. Herz, G. Schweizer, P. Hutzler, E. Glawischnig, R.A. Ruiz. The gene ENHANCER OF PINOID controls cotyledon development in the Arabidopsis embryo. Development 139 (2005), no. 18, 4063-4074.

[24] A. Volpert, Vit. Volpert, Vl. Volpert. Traveling wave solutions of parabolic systems. Translation of Mathematical Monographs, Vol. 140, Amer. Math. Society, Providence, 1994.

[25] M. Yamaguchi, H. Kato, S. Yoshida, S. Yamamura, H. Uchimiya, M. Umeda. Control of in vitro organogenesis by cyclin-dependent kinase activities in plants. Proc. Natl. Acad. Sci. USA, 100 (2003), no. 13, 8019-8023. 